\section{Genome Sequence Data of Leptosphaerulina arachidicola, a Causal Agent of Peanut Scorch Spot in China}

\author{
Yu-Ting Sheng, ${ }^{1,2}$ Xiao-Li Yu, ${ }^{3}$ Ting-Ting Mao, ${ }^{1,2}$ Juan Zhang, ${ }^{1,2}$ Xiao-Tong Guo, ${ }^{1,2}$ \\ Zhi-Zhong Song, ${ }^{1,2, \dagger}$ and Hong-Xia Zhang ${ }^{1,2, \dagger}$ \\ ${ }^{1}$ The Engineering Research Institute of Agriculture and Forestry, Ludong University, 186 \\ Hongqizhong Road, Yantai, China 264025 \\ ${ }^{2}$ Key Laboratory of Molecular Module-Based Breeding of High Yield and Abiotic Resistant Plants in \\ Universities of Shandong (Ludong University), 186 Hongqizhong Road, Yantai, Shandong Province, \\ China 264025 \\ ${ }^{3}$ Yantai Academy of Agricultural Sciences, 26 West Gangcheng Street, Yantai, Shandong Province, \\ China 2655000
}

\section{Abstract}

Peanut scorch spot caused by Leptosphaerulina arachidicola is one of the most severe leaf diseases of peanut that causes significant yield loss. Here, we report the first highquality genome sequence of $L$. arachidicola JB313 isolated from an infected peanut leaf in China. The genome size is $47.66 \mathrm{Mb}$, consisting of 65 scaffolds $\left(\mathrm{N}_{50}\right.$ length $\left.=1.58 \mathrm{Mb}\right)$ with a $\mathrm{G}+\mathrm{C}$ content of $49.05 \%$. The information in this report will provide a reference genome for future studies on the peanut scorch spot pathogen in peanut.

\section{Genome Announcement}

Peanut (Arachis hypogaea L.) is one of the most important oilseed crops commercially grown worldwide. Its total production and consumption in China rank first place globally, accounting for about $40 \%$ (Zhang et al. 2020). However, the grain yield and quality of peanut have been severely affected by peanut foliar fungal diseases. Peanut scorch spot, caused by Leptosphaerulina arachidicola, is one of the most common leaf diseases. Typically, this pathogen causes two types of symptoms, pepper spot and leaf scorch spot (Subrahmanyam and Smith 1991). The symptom of leaf scorch spot is more common than pepper spot. Usually, it occurs in combination with other leaf diseases, leading to the death of many leaves at the middle and late developmental stages of peanut.

The genus Leptosphaerulina was first reported by McAlpine in 1902 and, in total, 63 Leptosphaerulina members have been recorded in MycoBank (https://www.mycobank.org/). The causal pathogen of peanut scorch spot, $L$. arachidicola W. Y. Yen, M. J. Chen \& K. T. Huang (Yen et al. 1956), also known as L. crassiasca (Séchet) C. R. Jacks. \& D. K. Bell (Jackson and Bell 1968), belongs to the phylum Ascomycota, Pleosporales, Didymellaceae. It has been widely reported in peanut-growing regions such as Georgia (Luttrell and Boyle 1960), India (Nayudu 1963), the United States (Jackson and Bell 1969), South Africa (Young et al. 1980), Nepal (Pande et al. 1993), Brazil (Charchar et al. 2004), and Puerto Rico (Rosado-Rodríguez and Maldonado-Ramírez 2007). The pathogen survives in necrotic leaflets and becomes observable only at the sexual state with the generation of abundant ascospores. The strains of this pathogen isolated in Georgia and Taiwan can produce ascospores under light and dark conditions, respectively (Graham and Luttrell 1961), whereas the strain isolated in Henan Province of China didn't produce ascospores under either condition ( $\mathrm{Hu}$ et al. 2020), suggesting various mechanisms of ascospore production in this

${ }^{\dagger}$ Corresponding authors: Z.-Z. Song; szhzh2000@163.com, and H. Zhang; hxzhang@sibs.ac.cn
Funding

This work was jointly supported by the following grants: the Key Research and Development Project of Shandong Province (2020LZGC001), the National Key R\&D Program of China (2018YFD0201003), the National Natural Science Foundations of China (31601623), the Cooperation Project of University and Local Enterprise in Yantai of Shandong Province (2021XDRHXMPT09), and the Doctoral Fund of Shandong Natural Science Foundation (ZR2016CB19).

\section{Keywords}

genome, pathogen, peanut scorch spot

The author(s) declare no conflict of interest.

Accepted for publication 11 October 2021.

(c) 2022 The American Phytopathological Society 
Table 1. Genome features of Leptosphaerulina arachidicola JB313

\begin{tabular}{ll} 
Variables & \multicolumn{1}{c}{ Statistics } \\
Sequencing platform & PacBio + Illumina \\
PacBio long reads & $15.1 \mathrm{~Gb}$ \\
Illumina short reads & $7.1 \mathrm{~Gb}$ \\
Genome size & $47.66 \mathrm{Mb}$ \\
Sequencing coverage & $316.49 \times$ \\
Number of scaffolds & 65 \\
Average scaffold length & $0.73 \mathrm{Mb}$ \\
$\mathrm{N}_{50}$ of scaffold length & $1.58 \mathrm{Mb}$ \\
Maximum scaffold length & $2.4 \mathrm{Mb}$ \\
Scaffolds with telomere repeat & $9,13,15,21,23$ \\
$\quad$ (TTAGGG)n/(CCCTAA)n/both & \\
GC content & $49.05 \%$ \\
Number of predicted genes & 14,456 \\
Number of annotated genes & 11,716 \\
Number of secreted protein-coding genes & 1,047 \\
BUSCO completeness ${ }^{a}$ & $98.80 \%$ \\
Genome accession & $\mathrm{SRP} 332338$ \\
\hline
\end{tabular}

${ }^{a}$ Benchmarking universal single-copy ortholog.

pathogen. In addition, most isolates of $L$. arachidicola from different sources produced two types of symptoms, as described above (Subrahmanyam and Smith 1991). Some isolates survived as symptomless endophytes in the leaflets (Suryanarayanan and Murali 2006), suggesting that a potential variability of virulence may exist in this pathogen. However, because no genome resource has been reported in Leptosphaerulina spp., knowledge about the molecular basis of this pathogen is still limited. Accordingly, this work aims to provide a high-quality, whole-genome sequence of $L$. arachidicola for future studies on the lifestyle, host reorganization, and fungicide resistance of peanut scorch spot.

The $L$. arachidicola isolate JB313 was isolated from an infected peanut leaf in Yantai, Shandong Province, China. Pathogenicity assessment showed that JB313 is highly virulent on peanut leaves. Based on the DNA sequence of the internal transcribed spacer (ITS), JB313 is identified as $L$. arachidicola, using the primers ITS1 (5'-TCCGTAGGTGAACCTGCGG-3') and ITS4 (5'-TCCTCCGCTTATTGATA TGC-3'). Genomic DNA was extracted from mycelia cultured in potato dextrose broth at $25^{\circ} \mathrm{C}$ for 4 days using the Omega Fungal DNA Kit D3390-02 according to the manufacturer's instructions. Genome sequencing was performed using a combination of PacBio Sequel Single-Molecule Real Time and Illumina sequencing platforms at Shanghai Majorbio Bio-pharm Technology Co., Ltd. (Shanghai, China). The strain used to generate the genome sequence has been deposited in the herbarium of Ludong University, Yantai, China, which is publicly available upon requirement (accession number LDP01018).

In total, 1,109,531 PacBio long reads with $15.1 \mathrm{~Gb}$ of filtered subread bases and an average read length of $13 \mathrm{~kb}$, and 1,498,942 Illumina short reads containing $7.1 \mathrm{~Gb}$ bases, were generated. The PacBio reads were assembled into contigs using CANU (https://canu. readthedocs.io/en/latest/). The PacBio assembly results were further corrected for random errors using the Illumina clean reads. The final genome assembly size of $L$. arachidicola isolate JB313 was $47.66 \mathrm{Mb}$, consisting of 65 scaffolds, with a GC content of $49.05 \%$ (Table 1). The length of the longest scaffold was $2,401,293 \mathrm{bp}$, and the $\mathrm{N}_{50}$ length of all scaffolds was $1,578,034 \mathrm{bp}$. To evaluate the completeness of the assembly, benchmarking universal single-copy ortholog (BUSCO v3.0) (Simão et al. 2015) analyses of the draft genome sequences using "Ascomycota odb9" as a reference were performed. The results showed that 1,315 (98.6\%) complete BUSCOs were identified and the genome was well assembled. In total, 286 transfer RNA (tRNA) genes and 125 ribosomal RNA (rRNA) genes, including 18 of $28 \mathrm{~S}$ rRNA, 18 of $5.8 \mathrm{~S}$ rRNA, and 89 of $5 \mathrm{~S}$ rRNA, were identified using tRNAscan-SE (Lowe and Chan 2016) and Barrnap V0.8 (https://github.com/tseemann/barrnap/). The interspersed repeats of the genome assembly were predicted and annotated using RepeatMasker V4.0.7 (http://www.repeatmasker.org/). In total, 46 long terminal repeats, 303 long interspersed nuclear elements, 33 short interspersed nuclear elements, and 6 microsatellites were identified, with a $0.09 \%$ proportion of the assembled genome. Using the genome annotation and data management tool MAKER2 (Holt and Yandell 2011), 14,456 protein coding genes were 
identified in the genome assembly. The predicted genes constituted $61.22 \%$ of the assembled genome, with an average length of $2,018 \mathrm{bp}$. The predicted open reading frames were annotated through the database of NCBI nonredundant protein (14,456 genes, 100\%), Swiss-Prot (14,456 genes, 100\%) (Bairoch and Apweiler 2000), Pfam (9,480 genes, 65.6\%) (El-Gebali et al. 2019), gene ontology (6,842 genes, 47.3\%) (Ashburner et al. 2000), clusters of orthologous groups of proteins (11,716 genes, $81.0 \%$ ) (Tatusov et al. 2000), and Kyoto Encyclopedia of Genes and Genomes (3,501 genes, 24.2\%) (Kanehisa and Goto 2000) using sequence alignment tools BLAST, Diamond, and HMMER. Briefly, each set of query proteins was aligned with the databases, and annotations of best-matched subjects (E-value $<10^{-5}$ ) were obtained for gene annotation. The database of carbohydrate active enzymes (633 genes, 4.4\%) (Cantarel et al. 2009), P450 (390 genes, 2.7\%), pathogen-host interactions (1,401 genes, 9.7\%) (Urban et al. 2016), and database of fungal virulence factors (1,654 genes, $11.4 \%$ ) (Lu et al. 2012) were employed for further gene functional annotation. SignalP v4.0 (Petersen et al. 2011) prediction demonstrated that 1,047 proteins $(9.1 \%)$ contain signal peptide.

In summary, a high-quality and nearly complete genome resource of $L$. arachidicola was established. The draft genome sequence reported here has been deposited into the NCBI Sequence Read Archive (accession number SRP332338) under BioProject PRJNA753925, which is publicly accessible for future studies on peanut scorch spot.

\section{Literature Cited}

Ashburner, M., Ball, C. A., Blake, J. A., Botstein, D., Butler, H., Cherry, J. M., Davis, A. P., Dolinski, K., Dwight, S. S., Eppig, J. T., Harris, M. A., Hill, D. P., Issel-Tarver, L., Kasarskis, A., Lewis, S., Matese, J. C., Richardson, J. E., Ringwald, M., Rubin, G. M., and Sherlock, G. 2000. Gene ontology: Tool for the unification of biology. Nat. Genet. 25:25-29.

Bairoch, A., and Apweiler, R. 2000. The SWISS-PROT protein sequence database and its supplement TrEMBL in 2000. Nucleic Acids Res. 28:45-48.

Cantarel, B. L., Coutinho, P. M., Rancurel, C., Bernard, T., Lombard, V., and Henrissat, B. 2009. The Carbohydrate-Active EnZymes database (CAZy): An expert resource for glycogenomics. Nucleic Acids Res. 37:D233-D238.

Charchar, M., Anjos, J., Andrade, R., Fernandes, F. D., and Fernandes, C. D. 2004. Diseases of forage Arachis in Federal District, Brazil. Bol. Pesqui. Desenvolv. (Embrapa Cerrados) 133:5-18.

El-Gebali, S., Mistry, J., Bateman, A., Eddy, S. R., Luciani, A., Potter, S. C., Qureshi, M., Richardson, L. J., Salazar, G. A., Smart, A., Sonnhammer, E. L. L., Hirsh, L., Paladin, L., Piovesan, D., Tosatto, S. C. E., and Finn, R. D. 2019. The Pfam protein families database in 2019. Nucleic Acids Res. 47:D427D432.

Graham, J. H., and Luttrell, E. S. 1961. Species of Leptosphaerulina on forage plants. Phytopathology 51:680-693.

Holt, C., and Yandell, M. 2011. MAKER2: An annotation pipeline and genomedatabase management tool for second-generation genome projects. BMC Bioinf. 12:491.

Hu, Y., Cui, L., Gui, J., and Li, M. 2020. Identification and biological characteristics of the peanut scorch spot pathogen Leptosphaerulina arachidicola. J. Plant Prot. 47:221-222.

Jackson, C. R., and Bell, D. K. 1968. Leptosphaerulina crassiasca (Sechet) comb. nov., the cause of leaf scorch and pepper spot on peanut. Oleagineux 23:387388.

Jackson, C. R., and Bell, D. K. 1969. Diseases of peanut, groundnut, caused by fungi. Univ. Ga. Exp. Stn. Res. Bull. 56:7-15.

Kanehisa, M., and Goto, S. 2000. KEGG: Kyoto Encyclopedia of Genes and Genomes. Nucleic Acids Res. 28:27-30.

Lowe, T. M., and Chan, P. P. 2016. tRNAscan-SE On-line: Integrating search and context for analysis of transfer RNA genes. Nucleic Acids Res. 44:W54-W57.
Lu, T., Yao, B., and Zhang, C. 2012. DFVF: Database of fungal virulence factors. Database (Oxford) 2012:bas032.

Luttrell, B., and Boyle, L. 1960. Leaf spot of peanut in Georgia caused by Leptosphaerulina arachidicola. Plant Dis. Rep. 44:609-611.

Nayudu, M. V. 1963. Leptosphaerulina arachidicola on Groundnut. Indian Phytopathol. 16:384-386.

Pande, S., Sharma, B. P., Rao, G., Rao, J., Koirala, G., Rao, J. N., McDonald, D., and Joshi, M. 1993. First report of pepper spot and leaf scorch on groundnut in Nepal. Int. Arachis Newsl. 13:8-9.

Petersen, T. N., Brunak, S., Heijne, G. V., and Nielsen, H. H. 2011. SIGNALP 4.0: Discriminating signal peptides from transmembrane regions. Nat. Methods 8 : 785-786.

Rosado-Rodríguez, G., and Maldonado-Ramírez, S. 2007. First report of Leptosphaerulina crassiasca (Ascomycota) in Puerto Rico. J. Agric. Univ. P. R. 91:47-48.

Simão, F., Waterhouse, R. M., Panagiotis, I., Kriventseva, E. V., and Zdobnov, E. M. 2015. BUSCO: Assessing genome assembly and annotation completeness with single-copy orthologs. Bioinformatics 31:3210-3212.

Subrahmanyam, P., and Smith, D. H. 1991. Variability in pathogenicity and symptomatology of Leptosphaerulina crassiasca on peanut. Plant Dis. 75:1266-1269.

Suryanarayanan, T. S., and Murali, T. S. 2006. Incidence of Leptosphaerulina crassiasca in symptomless leaves of peanut in southern India. J. Basic Microbiol. 46:305-309.

Tatusov, R. L., Galperin, M. Y., Natale, D. A., and Koonin, E. V. 2000. The COG database: A tool for genome-scale analysis of protein functions and evolution. Nucleic Acids Res. 28:33-36.

Urban, M., Cuzick, A., Rutherford, K., Irvine, A., and Hammond-Kosack, K. E. 2016. PHI-base: A new interface and further additions for the multi-species pathogen-host interactions database. Nucleic Acids Res. 45:D604-D610.

Yen, J., Chen, M. J., and Huang, K. T. 1956. Leaf scorch of peanut (a new disease). J. Agric. For. 10:1-24

Young, B. W., Blamey, F., and Chapman, J. 1980. Studies on the occurrence, epidemiology and control of leaf and stem diseases of groundnuts. Tech. Commun. Dep. Agric. Tech. Serv. Repub. S. Afr. No. 166.

Zhang, H. F. X., Wang, L., Li, G., Qiao, L., Wei, D., and Zheng, G. 2020. Establishing a peanut big data platform in China: A proposal and applications. $J$ Agric Big Data 2:45-52. 